TecnoLógicas

ISSN 0123-7799

ISSN-e 2256-5337

Vol. 20, No. 40, pp. 53-69

Sep-dic de 2017

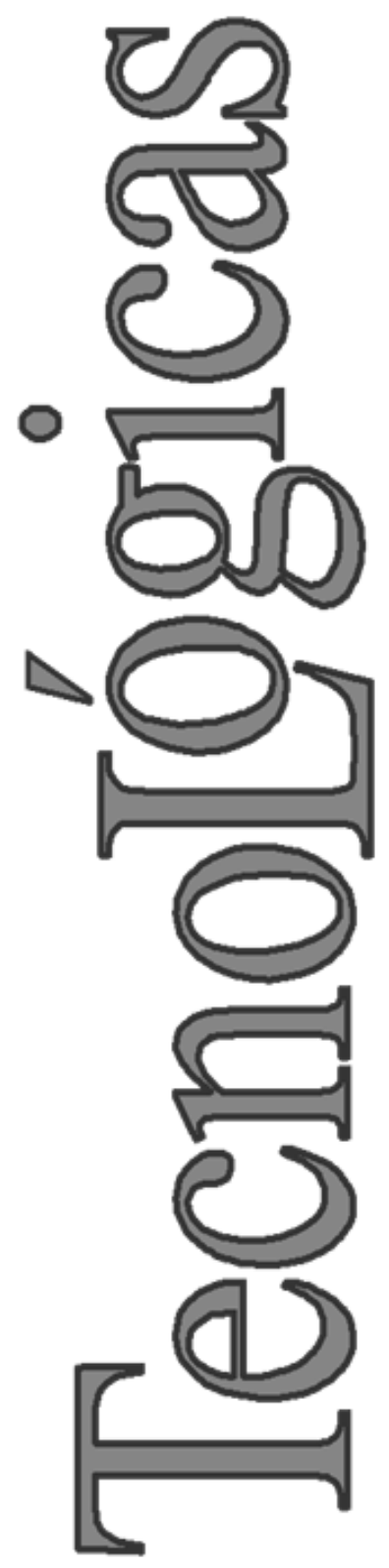

(C) Copyright 2015 por autores y Tecno Lógicas Este trabajo está licenciado bajo una Licencia Internacional Creative Commons Atribución (CC BY)

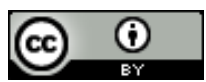

\section{Aislamiento térmico de tuberías de acero que transportan fluidos calientes a partir de recubrimientos elaborados mediante proyección térmica}

\section{Thermal isolation of steel pipelines employed in the transportation of hot fluids from thermal sprayed coatings}

\author{
Daniel García-Muñoz y Fabio Vargas-Galvis ${ }^{2}$
}

Recibido: 22 de noviembre de 2016

Aceptado: 12 de junio de 2017

Cómo citar / How to cite

D. García-Muñoz y F. Vargas-Galvis, Aislamiento térmico de tuberías de acero que transportan fluidos calientes a partir de recubrimientos elaborados mediante proyección térmica. TecnoLógicas, vol. 20, no. 40, pp. 53-69, 2017.

Estudiante de Ingeniería de Materiales, Grupo GIMACYR, Universidad de Antioquia, Medellín-Colombia, daniel.garciam@udea.edu.co

2 PhD. en Ingeniería de Materiales Cerámicos y Tratamiento de Superficies, Grupo GIMACYR, Universidad de Antioquia, Medellín-Colombia, fabio.vargas@udea.edu.co 


\section{Resumen}

Se depositaron recubrimientos cerámicos, a partir de polvos comerciales de $\mathrm{Al}_{2} \mathrm{O}_{3}, \mathrm{Al}_{2} \mathrm{O}_{3}$ $\mathrm{TiO}_{2}$, y $\mathrm{Al}_{2} \mathrm{O}_{3}-\mathrm{ZrO}_{2}$ sobre la superficie exterior de tuberías de acero al carbono ASTM A106 Grado B con y sin capa base de una aleación de níquel, con el fin de reducir las pérdidas de calor contenido en fluidos calientes que son transportados a través de este tipo de ductos. Tanto la capa base como los recubrimientos cerámicos fueron depositados mediante proyección térmica oxiacetilénica y posteriormente se evaluó la transferencia de calor en estado transitorio, para lo cual se introdujo en las tuberías aceite de silicona calentado a $160^{\circ} \mathrm{C}$. La variación de temperatura con el tiempo del aceite de silicona y de la pared externa de cada tubo fue medida, encontrando que además de la baja conductividad térmica de los materiales cerámicos, un mayor espesor y una mayor porosidad del recubrimiento brindan medios efectivos de aislamiento térmico, y que la capa base actúa como una resistencia térmica que consume calor y contribuye a la disminución de la temperatura superficial de la tubería.

\section{Palabras clave}

Proyección térmica, barreras térmicas, tuberías, líquidos calientes, capa base.

\section{Abstract}

Ceramic coatings of $\mathrm{Al}_{2} \mathrm{O}_{3}, \mathrm{Al}_{2} \mathrm{O}_{3}-\mathrm{TiO} 2$, and $\mathrm{Al}_{2} \mathrm{O}_{3}-\mathrm{ZrO}_{2}$ commercial powders were thermally sprayed on the external surface of ASTM A106 Grade-B carbon steel pipelines (with and without a bond coat of a commercial nickel alloy) to reduce the loss of heat contained in hot fluids that are transported through this kind of ducts. The bond coat, as well as the ceramic coatings, were deposited by means of oxy-acetylene thermal spray; afterwards, the heat transfer in transitory state was evaluated by placing heated silicone oil at $160^{\circ} \mathrm{C}$ inside the pipelines. The temperature variation over time of the silicone oil and the external wall of each pipeline was measured. Besides the low thermal conductivity of the ceramic materials, a thicker and more porous coating provides a more effective means for thermal isolation. Additionally, the bond coat acts as a thermal resistance, which consumes heat and contributes to the reduction of the superficial temperature of the pipeline.

\section{Keywords}

Thermal spray, barrier coatings, pipelines, hot fluids, bond coat. 
Aislamiento térmico de tuberías de acero que transportan fluidos calientes a partir de recubrimientos elaborados mediante proyección térmica

\section{INTRODUCCIÓN}

En la industria petrolera es fundamental la disposición de redes de tuberías a lo largo de vastas extensiones de territorio para el transporte del crudo entre los sitios de extracción, refinación y centros de almacenamiento o puertos de exportación, por lo que estas tuberías atraviesan gran variedad de suelos, climas y en general, diferentes condiciones de operación.

Debido a la alta viscosidad de algunos crudos, es necesario aumentar su temperatura (en ocasiones hasta $100^{\circ} \mathrm{C}$ ) en las zonas cercanas a la boca del pozo de extracción y en trayectos de hasta 1 kilómetro, para facilitar el bombeo a través de las tuberías, las cuales están fabricadas en acero al carbono, material que presenta alta transferencia de calor $(48.9 \mathrm{~W} / \mathrm{mK})$ [1] $\mathrm{y}$, por lo tanto, una buena cantidad de la energía aportada al fluido es perdida a través del ducto. Adicionalmente, una parte de la longitud de dichas tuberías se encuentra enterrada en suelos que pueden ser desde ácidos hasta alcalinos. Esto deriva no solo en problemas corrosivos de las tuberías, sino en afectaciones a los suelos e incluso al agua del nivel freático en zonas circundantes, generando impactos ambientales negativos.

De acuerdo con lo anterior, se requiere de sistemas de recubrimiento que además de reducir la pérdida de calor desde el interior de la tubería, la proteja de los efectos corrosivos del suelo o del ambiente y que, además, resista las condiciones mecánicas impuestas tanto por el suelo (rayaduras por agregados del suelo $u$ otros), como durante su operación y mantenimiento.

Con el fin de resolver el problema de aislamiento térmico han sido utilizados diversos sistemas, entre los cuales se encuentran pinturas, espumas poliméricas, camisas cerámicas y lanas minerales, cada uno con diferentes tasas de transferencia de calor en función del espesor del sistema de aislamiento. Algunos de ellos no cumplen con las condiciones de operación mínimas, bien sea por sus bajas propiedades mecánicas o poca efectividad de aislamiento. Un ejemplo puntual es el uso de lana de vidrio recubierta con una capa de aluminio, el cual es un sistema que presenta un deterioro prematuro a causa de las infiltraciones de agua que pueden presentarse hasta alcanzar la tubería, originando problemas corrosivos además de pérdidas de calor [2], [3].

La mala elección de un sistema de aislamiento conlleva a invertir recursos adicionales para controlar las pérdidas de energía, a las cuales deben sumarse inspecciones con mayor frecuencia y mantenimientos, en el peor de los casos. Se busca entonces que dichos medios aislantes tengan bajos coeficientes de conductividad térmica y que además sean de bajo espesor, pues mientras más delgados sean habrá menor área superficial expuesta a la emisión de calor [4], [5].

Es sabido que los materiales cerámicos presentan generalmente bajos coeficientes de transferencia de calor, alta dureza y buena resistencia al desgaste abrasivo y a la corrosión [6].

En cuanto a soluciones encontradas en la literatura que aborden este tipo de problemas desde un enfoque principalmente térmico, pero además mecánico y químico, se ha encontrado que los sistemas de recubrimiento de barrera térmica satisfacen los requerimientos adecuadamente.

Por lo general, se consideran barreras térmicas a aquellas capas cerámicas de espesor generalmente inferior a $300 \mu \mathrm{m}$, cuya conductividad térmica es menor a 2 W/m.K, y que son depositadas sobre una capa de anclaje constituida a partir de una aleación de níquel de espesor inferior a 200 $\mu \mathrm{m}$ [7]. 
Aislamiento térmico de tuberías de acero que transportan fluidos calientes a partir de recubrimientos elaborados mediante proyección térmica

Tabla 1. Propiedades térmicas del sustrato y de materiales potencialmente útiles como barrera térmica. Fuente: autores.

\begin{tabular}{llll}
\hline & $\mathbf{T}^{\circ}$ fusión $\left[{ }^{\circ} \mathbf{C}\right]$ & $\mathbf{A}^{(\mathbf{1})}\left[\boldsymbol{\mu m} / \mathbf{m}^{\circ} \mathbf{C}\right.$ & $\mathbf{k}[\mathbf{W} / \mathbf{m K}]$ \\
\hline $100 \% \mathrm{Ni}[1]$ & 1455 & 13,1 & 60,7 \\
Alúmina alfa [1], [20] & 2054 & 5,5 & $32,3-46$ \\
$\mathrm{Al} 2 \mathrm{O} 3 \approx 40 \% \mathrm{TiO} 2[14],[15]$ & 1840 & $0.8 \times 10-8$ & 1.5 \\
$\mathrm{Al} 2 \mathrm{O} 3 \approx 60 \% \mathrm{ZrO2}[14]$ & 2054 & 8,1 & 21,6 \\
Acero al carbono [1], [16] & 1515 & 11,7 & 48,9 \\
\hline
\end{tabular}

(1) Valores a temperatura entre 20 y $120^{\circ} \mathrm{C}$

Su diseño en cuanto a materiales, número de capas, espesor, porosidad y técnica de aplicación, se encuentra completamente ligado al entorno al que será expuesta la pieza en cuestión, es decir, que esta delgada capa como todo recubrimiento cerámico, podrá estar expuesta a ambientes corrosivos, con amplios rangos de variación en temperaturas, medios líquidos o gaseosos, entre otros, que pondrán a prueba su integridad y durabilidad en el tiempo.

La capa base en las barreras térmicas cumple una función triple: mejoramiento de la adhesión entre el sustrato y la capa cerámica, proteger el sustrato contra la corrosión y oxidación y reducción de los esfuerzos causados por la diferencia de coeficientes de dilatación térmica entre la capa cerámica y el sustrato [7].

La capa base es utilizada en sistemas que utilizan una capa superior cerámica que serán expuestos a ciclos térmicos (aunque ellos se realicen en rangos de temperaturas tan bajas como entre la temperatura ambiente y $100{ }^{\circ} \mathrm{C}$ ) como aquellos a los que operan normalmente las tuberías de transporte de hidrocarburos altamente viscosos. De acuerdo con lo anterior, los sistemas de recubrimiento constituidos por una aleación de níquel, que actúa como capa base y de una capa superior cerámica, pueden alcanzar los requerimientos térmi cos y mecánicos solicitados a las tuberías de acero al carbono que transportan fluidos calientes, de acuerdo con las propiedades reportadas en la Tabla 1 [8], [9],[10], [11].

En el proceso de proyección térmica mediante combustión, las partículas utilizadas para la elaboración del recubrimien- to son fundidas total o parcialmente en una llama oxiacetilénica, y propulsadas a lo largo de dicha llama hacia el sustrato donde se apilan entre ellas. El recubrimiento es entonces formado como resultado de un acomodamiento en capas o lamelles, de pequeños discos aplanados también conocidos como splats [12] los cuales se adhieren principalmente por anclaje mecánico sobre un sustrato con superficie limpia y de rugosidad controlada [13].

Con base en lo anterior, en este trabajo se depositó sobre tuberías de acero al carbono ASTM A106 Grado B una aleación de níquel y sobre ella tres diferentes materiales cerámicos, con el fin de evaluar su capacidad para retener el calor de un fluido contenido en su interior. Además del efecto de la aleación de níquel utilizada como capa base, se evaluó el efecto de la composición química del recubrimiento cerámico, de su espesor y porosidad.

\section{METODOLOGÍA EXPERIMENTAL}

\subsection{Materiales}

Para la elaboración de los sistemas de aislamiento térmico se utilizaron polvos cerámicos comerciales de $\mathrm{Al}_{2} \mathrm{O}_{3}$ referencia Sultzer-Metco ${ }^{\mathrm{TM}} 105 \mathrm{SFP}$, de $\mathrm{Al}_{2} \mathrm{O}_{3} \approx 40 \%$ en peso de $\mathrm{TiO}_{2}$ referencia Saint Gobain $108^{\mathrm{TM}}$, y $\mathrm{Al}_{2} \mathrm{O}_{3} \approx 60 \%$ en peso de $\mathrm{ZrO}_{2}$ referencia Eutectic Castolin ${ }^{\mathrm{TM}}$ 25088. Para la capa base se utilizó el polvo comercial Eutectic Castolin ${ }^{\mathrm{TM}}$ CPM 1205, correspondiente a una aleación de níquel con aproximadamente $1,8 \%$ en peso de silicio. Los 
Aislamiento térmico de tuberías de acero que transportan fluidos calientes a partir de recubrimientos elaborados mediante proyección térmica

sistemas de recubrimiento depositados son descritos en Tabla (2). Los tubos de acero al carbono utilizados como sustrato fueron recibidos con una pintura orgánica de color negro en la superficie externa, la cual fue codificada como (M-I) y evaluada de la misma forma que los recubrimientos depositados mediante proyección térmica. La muestra M-0 corresponde a la tubería sin recubrimiento alguno en la superficie.

La composición química de los materiales depositados fue determinada mediante Fluorescencia de Rayos X (FRX) por longitud de onda dispersiva utilizando un espectrómetro marca Thermo, referencia Optimi’x.

\subsection{Preparación superficial}

La superficie exterior de los tubos fue limpiada con un chorro abrasivo de partí- culas de corindón hasta obtener una rugosidad media $(\mathrm{Ra})$ entre 5 y $7 \mu \mathrm{m}$, la cual fue medida con un rugosímetro marca Mitutoyo referencia SJ 201.

\subsection{Recubrimientos}

Los recubrimientos, tanto de la capa base como para las capas cerámicas, fueron depositados utilizando una antorcha de combustión oxiacetilénica marca EutecticCastolin, referencia TeroDyn 2000, la cual ha sido modificada e incorporada a la cámara Areste 1 del grupo GIPIMME de la Universidad de Antioquia. Esta cámara cuenta con sistemas electromecánicos y un sensor óptico de temperatura, para controlar las principales variables del proceso de proyección térmica. En la Fig. 1 se muestran los tubos recubiertos en la superficie exterior.

\begin{tabular}{ccc} 
Tabla 2. Sistemas de recubrimiento depositados para análisis de transferencia de calor. Fuente: autores \\
\hline Código de la muestra & Capa base & Recubrimiento cerámico \\
\hline M-I & Pintura & - \\
M0-A & - & - \\
M0-B & Aleación base níquel & - \\
M1-A & - & $\mathrm{Al}_{2} \mathrm{O}_{3} \approx 40 \% \mathrm{TiO}_{2}$ \\
M1-B & Aleación base níquel & $\mathrm{Al}_{2} \mathrm{O}_{3} \approx 40 \% \mathrm{TiO}_{2}$ \\
M2-A & - & $\mathrm{Alú}_{\text {mina }}$ \\
M2-B & Aleación base níquel & $\mathrm{Alúmina}$ \\
M3-A & - & $\mathrm{Al}_{2} \mathrm{O}_{3} \approx 60 \% \mathrm{ZrO}_{2}$ \\
M3-B & Aleación base níquel & $\mathrm{Al}_{2} \mathrm{O}_{3} \approx 60 \% \mathrm{ZrO}_{2}$ \\
\hline
\end{tabular}

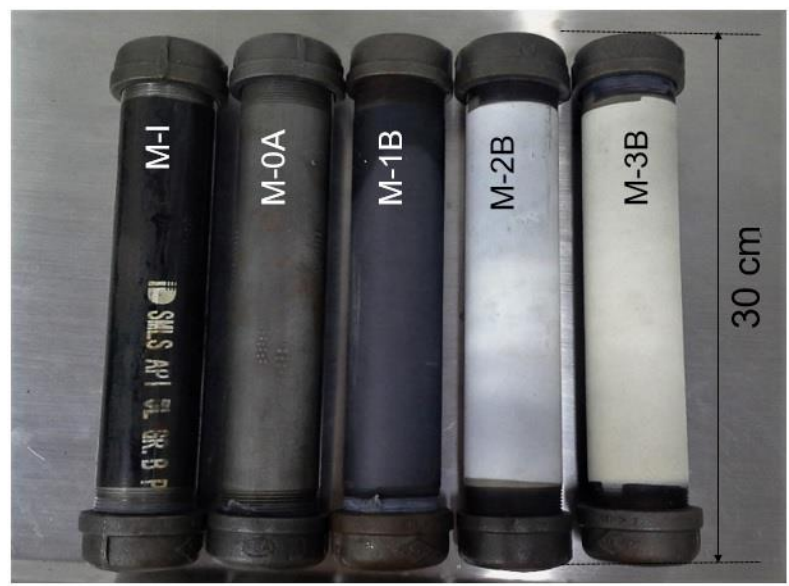

Fig. 1 Tubos recubiertos superficialmente. Fuente: autores. 
Aislamiento térmico de tuberías de acero que transportan fluidos calientes a partir de recubrimientos elaborados mediante proyección térmica

Los parámetros de proyección presentados en la Tabla 3, corresponden a los empleados para la elaboración de la capa base y de las capas cerámicas, los cuales fueron ajustados de manera que se obtuvieran recubrimientos con espesores inferiores a $250 \mu \mathrm{m}$ y $400 \mu \mathrm{m}$ respectivamente. Estos parámetros fueron escogidos de acuerdo a trabajos previos reportados por A. González[17], y con experiencias previas de trabajo en el grupo GIMACYR, como las reportadas por J. E. Rodríguez[18].

\subsection{Caracterización de los recubrimientos}

El espesor de los recubrimientos realizados fue medido por diferencia entre el diámetro del sustrato antes y después del recubrimiento. Las medidas se hicieron con un calibrador digital marca Mitutoyo, el cual cuenta con una resolución de $10 \mu \mathrm{m}$.

Las fases cristalinas de los recubrimientos cerámicos se detectaron mediante Difracción de Rayos X (DRX) en un equipo marca Empyrean de radiación $\mathrm{Cu}-\mathrm{Ka}$ con longitudes de onda $\lambda_{1}=1.540598 \AA$ y $\lambda_{2}=1.544426 \AA$, con ángulos de barrido entre $10^{\circ}$ y $70^{\circ}$. El análisis de los patrones de difracción se realizó con el software X'Pert HighScore Plus de la firma PANalytical.

Por su parte, el porcentaje de porosidad presente en la estructura de cada uno de los recubrimientos cerámicos se determinó utilizando el software de uso libre ImageJ, a partir de imágenes tomadas mediante microscopía óptica siguiendo los lineamien- tos de la norma ASTM E2109 [15], para lo cual la sección transversal de los recubrimientos fue preparada según lo establecido en la norma ASTM E1920 [19].

\subsection{Pruebas térmicas en estado transitorio}

Para evaluar la capacidad de aislamiento de los sistemas planteados, se introdujo en cada probeta tubular aceite de silicona calentado a una temperatura de $160^{\circ} \mathrm{C}$ y se midió la variación de la temperatura tanto del aceite, como de la superficie exterior del tubo a intervalos de un minuto durante media hora. La temperatura del aceite fue medida con un termómetro, mientras que la temperatura exterior del tubo fue medida con una termocupla acoplada a un multímetro digital marca Fluke referencia 179, los dos previamente calibrados y con una sensibilidad de $1^{\circ} \mathrm{C}$. Ver Fig. 2. La temperatura del aceite de silicona fue medida en el centro de la zona que fue llenada con este fluido, y la de la tubería fue medida a la misma altura en que se realizó la medida del aceite de silicona, pero sobre la pared externa del tubo. Para reducir las pérdidas de calor y evitar la fuga del aceite de silicona, se sellaron los extremos de cada tubo hasta donde fueron llenados con el fluido, utilizando tapones de teflón, uno de los cuales permite la inserción del termómetro. A la prueba se realizó un duplicado evidenciando una buena reproducibilidad.

\begin{tabular}{lcc}
\multicolumn{3}{c}{ Tabla 3. Parámetros de proyección para las capas base y cerámicas. Fuente: autores. } \\
\hline & Capa base & Capa cerámica \\
\hline Distancia de proyección (cm) & 13 & 9 \\
Flujo de gases (L/min) (Acetileno: Oxígeno) & $29: 35$ & $22: 92$ \\
Presión de aire (psi) & 30 & 30 \\
Pases de precalentamiento & 2 & 3 \\
Pases de proyección & 4 & 5 \\
Velocidad vertical de antorcha $(\mathrm{cm} / \mathrm{s})$ & 0,73 & 0,73 \\
Velocidad de portamuestras $(\mathrm{rpm})$ & 82 & 116 \\
\hline
\end{tabular}


Aislamiento térmico de tuberías de acero que transportan fluidos calientes a partir de recubrimientos elaborados mediante proyección térmica

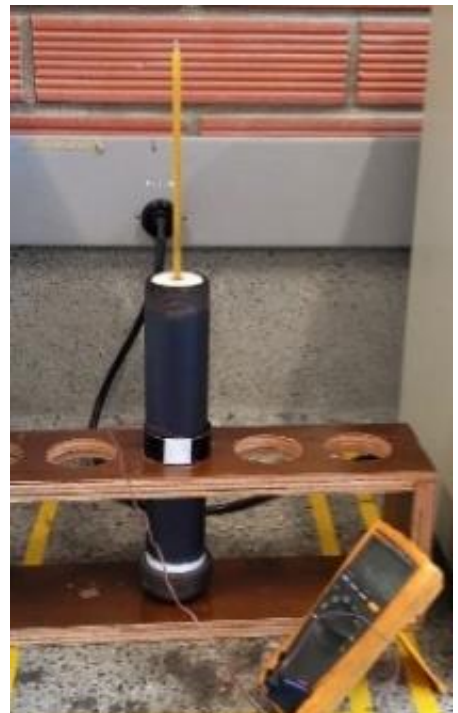

Fig. 2 Montaje realizado para las pruebas de transferencia de calor en estado transitorio. Fuente: autores.

\section{RESULTADOS Y DISCUSIÓN}

\subsection{Caracterización de los materiales}

Los resultados del análisis químico realizado a cada uno de los polvos utilizados para la elaboración de los recubrimientos son mostrados en Tabla (4).

En la tabla el ítem "otros" corresponde a óxidos detectados en cada material de partida en cantidades inferiores a $0,1 \%$ en peso. De acuerdo con la tabla anterior, se pudo establecer que los polvos seleccionados son acordes en composición química a lo especificado por el fabricante.

En la tabla anterior el ítem "otros" corresponde a óxidos detectados en cada material de partida en cantidades inferiores a $0,1 \%$ en peso. De acuerdo con la tabla anterior, se pudo establecer que los polvos seleccionados son acordes en composición química a lo especificado por el fabricante.

Por su parte, el análisis morfológico de las partículas indicó que los polvos de la aleación de níquel y de la mezcla cerámica de $\mathrm{Al}_{2} \mathrm{O}_{3} \approx 60 \%$ en peso de $\mathrm{ZrO}_{2}$ poseen alta esfericidad, mientras que aquellos de alúmina y de $\mathrm{Al}_{2} \mathrm{O}_{3} \approx 40 \%$ en peso de $\mathrm{TiO}_{2}$ son de morfología irregular y con aristas agu- das que son características del proceso de molienda al que son sometidos para ajustar su tamaño. Además se evidenció que los polvos de $\mathrm{Al}_{2} \mathrm{O}_{3} \approx 60 \%$ en peso de $\mathrm{ZrO}_{2}$ están constituidos por múltiples partículas de orden nanométrico y submicrométrico que generalmente son aglomeradas por procesos como el de secado por aspersión (en inglés Spray Drying).

Las partículas metálicas esféricas son obtenidas a partir de procesos de fusión y posterior atomización en un líquido o un gas para su rápido enfriamiento. En general, el tamaño de todos los materiales proyectados son de orden micrométrico (d10> $5 \mu \mathrm{m}$ y d9o $65 \mu \mathrm{m}$ ), incluso las partículas nanométricas y submicrométicas aglomeradas. Ver Fig 3.

\subsection{Sustratos}

Los valores rugosidad aritmética media (Ra) obtenidos para las probetas se presentan en Tabla (5).

\begin{tabular}{c}
\multicolumn{2}{l}{$\begin{array}{l}\text { Tabla 5. Valores de rugosidad obtenidos sobre la su- } \\
\text { perficie de los sustratos. Fuente autores }\end{array}$} \\
\begin{tabular}{cc} 
Código de muestra & Rugosidad $\mu \mathrm{m}(\mathrm{Ra})$ \\
\hline M0-A & $5,96 \pm 0,94$ \\
M0-B & $6,64 \pm 0,52$ \\
M1-A & $5,34 \pm 0,51$ \\
M1-B & $7,10 \pm 0,95$ \\
M2-A & $42 \pm 0,27$ \\
M2-B & $7,59 \pm 0,69$ \\
M3-A & $5,08 \pm 0,45$ \\
M3-B & $6,87 \pm 0,53$
\end{tabular}
\end{tabular}

Buscando asegurar un buen anclaje mecánico entre el sustrato y los depósitos en cuestión, se optó por alcanzar valores de rugosidad superficial que fueran iguales o superiores a un Ra de $5 \mu \mathrm{m}$. Algunos autores como M. Mellali et al [13] aseguran que la limpieza y la rugosidad del sustrato son fundamentales para una buena adherencia en especial para sustratos sin precalentamiento al momento de depositar el recubrimiento, pero un Ra excesivo puede generar decremento en la adhesión. 
Aislamiento térmico de tuberías de acero que transportan fluidos calientes a partir de recubrimientos elaborados mediante proyección térmica
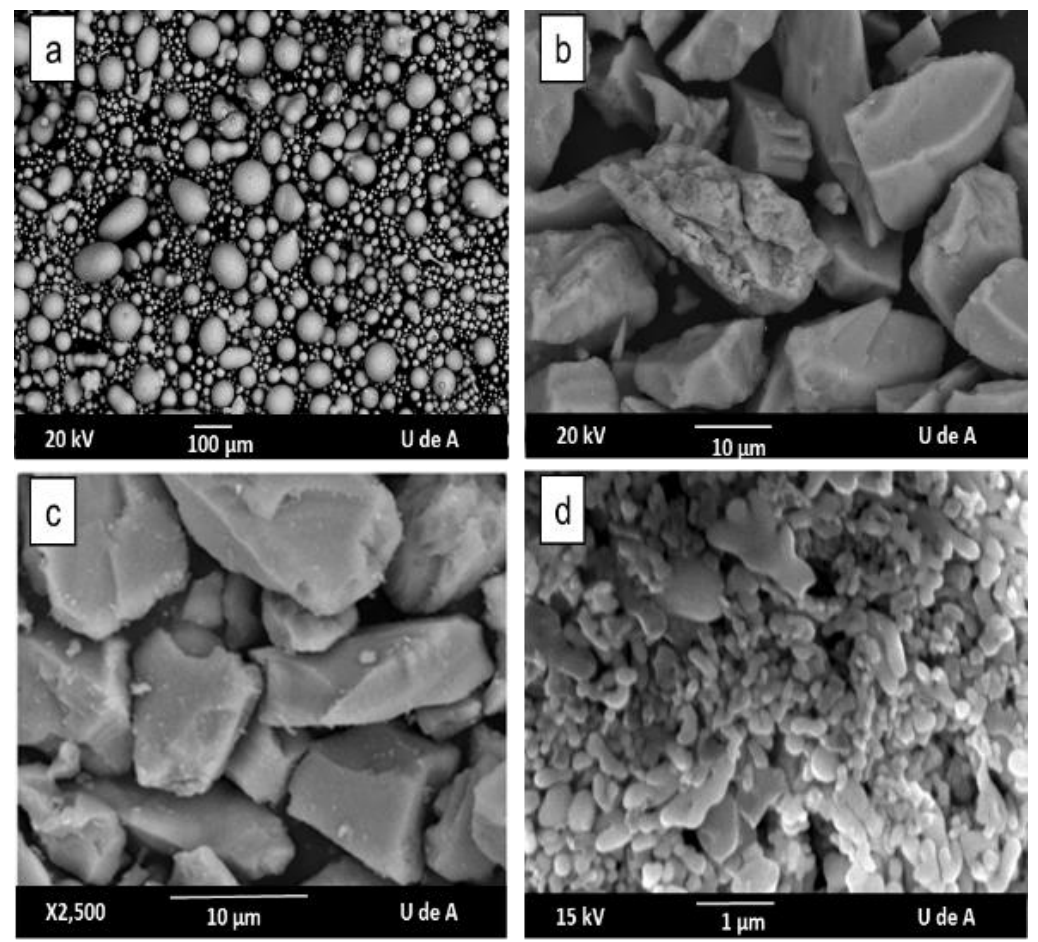

Fig. 3 Morfología de los materiales de partida. (a) Eutectic Castolin CPM 1205, (b) Saint Gobain 108, (c) Sulzer-Metco 105 SFP, (d) Eutectic Castolin 25088. Fuente: autores.

Tabla 4. Composición química de los polvos de partida. Fuente: autores.

\begin{tabular}{|c|c|c|c|}
\hline Firma & Referencia & \multicolumn{2}{|c|}{ Composición (\%Wt) } \\
\hline \multirow{4}{*}{$\begin{array}{l}\text { Eutectic Casto- } \\
\text { lin }\end{array}$} & \multirow{4}{*}{ CPM 1205} & $\mathrm{Ni}$ & 97,6 \\
\hline & & $\mathrm{Si}$ & 1,79 \\
\hline & & $\mathrm{Fe}$ & 0,34 \\
\hline & & $\mathrm{Al}$ & 0,18 \\
\hline Otros & & & 0,09 \\
\hline \multirow{5}{*}{ Saint Gobain } & \multirow{5}{*}{108} & $\mathrm{Al} 2 \mathrm{O} 3$ & 50,27 \\
\hline & & $\mathrm{TiO} 2$ & 47,68 \\
\hline & & $\mathrm{ZrO} 2$ & 0,66 \\
\hline & & $\mathrm{V} 2 \mathrm{O} 5$ & 0,60 \\
\hline & & $\mathrm{Fe} 2 \mathrm{O} 3$ & 0,25 \\
\hline Otros & & & 0,54 \\
\hline \multirow{3}{*}{ Sulzer-Metco } & \multirow{3}{*}{$105 \mathrm{SFP}$} & $\mathrm{Al} 2 \mathrm{O} 3$ & 99,71 \\
\hline & & $\mathrm{SiO} 2$ & 0,17 \\
\hline & & $\mathrm{Fe} 2 \mathrm{O} 3$ & 0,03 \\
\hline Otros & & & 0,09 \\
\hline \multirow{5}{*}{$\begin{array}{l}\text { Eutectic Casto- } \\
\text { lin }\end{array}$} & \multirow{5}{*}{25088} & $\mathrm{ZrO} 2$ & 67,03 \\
\hline & & $\mathrm{Al} 2 \mathrm{O} 3$ & 31,05 \\
\hline & & $\mathrm{HfO} 2$ & 0,95 \\
\hline & & $\mathrm{Y} 2 \mathrm{O} 3$ & 0,23 \\
\hline & & $\mathrm{SiO} 2$ & 0,21 \\
\hline Otros & & & 0,53 \\
\hline
\end{tabular}

[60] TecnoLógicas, ISSN 0123-7799, ISSN-e 2256-5337, Vol. 20, No. 40, sep-dic de 2017, pp. 53-69 
Aislamiento térmico de tuberías de acero que transportan fluidos calientes a partir de recubrimientos elaborados mediante proyección térmica

\subsection{Recubrimientos}

\subsubsection{Espesor}

Los espesores medidos para cada uno de los recubrimientos se muestran en la Tabla 6

\subsubsection{Sustratos}

Los espectros de difracción de rayos $\mathrm{X}$ obtenidos para las muestras con capa base M1-B, M2-B y M3-B se observan en las Fig. 4,5 y 6 respectivamente.

A partir del espectro de la Fig. 4 correspondiente al recubrimiento de $\mathrm{Al}_{2} \mathrm{O}_{3}-\mathrm{TiO}_{2}$, se pudo determinar que las fases cristalinas que constituyen este recubrimiento son los titanatos de aluminio ( $\left.\mathrm{Al}_{2} \mathrm{TiO}_{5}\right)$ marcados como 1 en el espectro (Código de referencia ICDD 01-070-1434) y los tridecaóxido dititanio de hexaaluminio $\left(\mathrm{Al}_{12} \mathrm{Ti}_{4} \mathrm{O}_{26}\right)$ marcados como 2 (Código de referencia COD 96-201-4755). Ambas son fases ortorrómbicas caracterizadas por su baja conductividad térmica $(\mathrm{k}=1.5 \mathrm{~W} / \mathrm{mK})$ [14], resistencia a los choques térmicos y muy bajos coeficientes de dilatación térmica, lo que le da estabilidad en temperaturas de hasta $750^{\circ} \mathrm{C}$, donde empiezan a descomponerse cuando no están dopados [18].

El espectro de la Fig.5 corresponde al recubrimiento de alúmina, en el cual se identifica a la $\mathrm{Al}_{2} \mathrm{O}_{3}-\mathrm{a}$ marcada como 1 (Código de referencia COD 96-900-9684) y la $\mathrm{Al}_{2} \mathrm{O}_{3}-\mathrm{Y}$ marcada como 2 (Código de referencia ICDD 00-029-0063 e ICDD 00004-0858), como las fases cristalinas que lo constituyen. En recubrimientos elaborados mediante proyección térmica la $\mathrm{Al}_{2} \mathrm{O}_{3}-\mathrm{a}$ está asociada a una fase remanente que estaba presente en los polvos de partida y que no se transforma durante la proyección térmica, cuando las partículas de alúmina se funden solo en la superficie y su núcleo permanece sólido y sin mayores transformaciones de las fases. Generalmente la estructura de los recubrimientos con partículas parcialmente fundidas tiene alta porosidad, lo cual es favorable para el caso de aislamientos térmicos. Por su parte, la $\mathrm{Al}_{2} \mathrm{O}_{3}-\mathrm{Y}$ es una fase meta estable producida a partir de la solidificación rápida de partículas fundidas de alúmina. Esta fase, por encima de $700^{\circ} \mathrm{C}$ puede sufrir transformaciones a $\mathrm{Al}_{2} \mathrm{O}_{3}-\alpha$ pasando previamente por una fase delta [20].

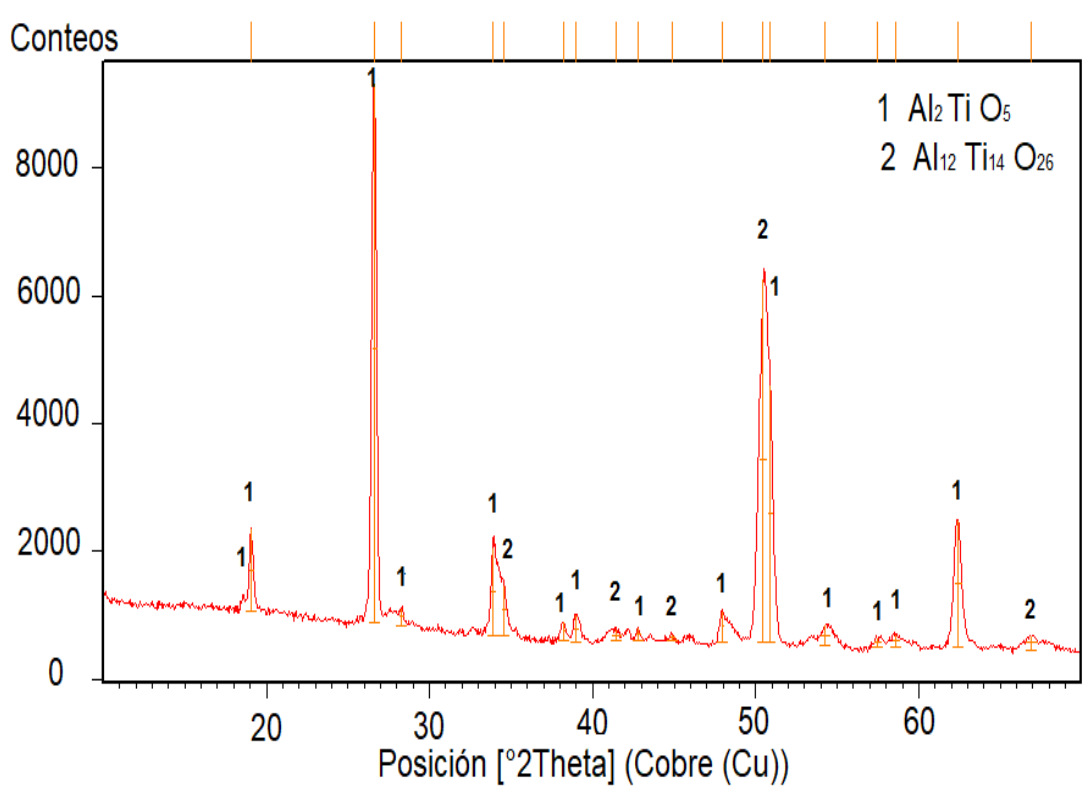

Fig. 4 Espectro de Difracción de Rayos X para la muestra M1-B. Fuente: autores 
Aislamiento térmico de tuberías de acero que transportan fluidos calientes a partir de recubrimientos elaborados mediante proyección térmica

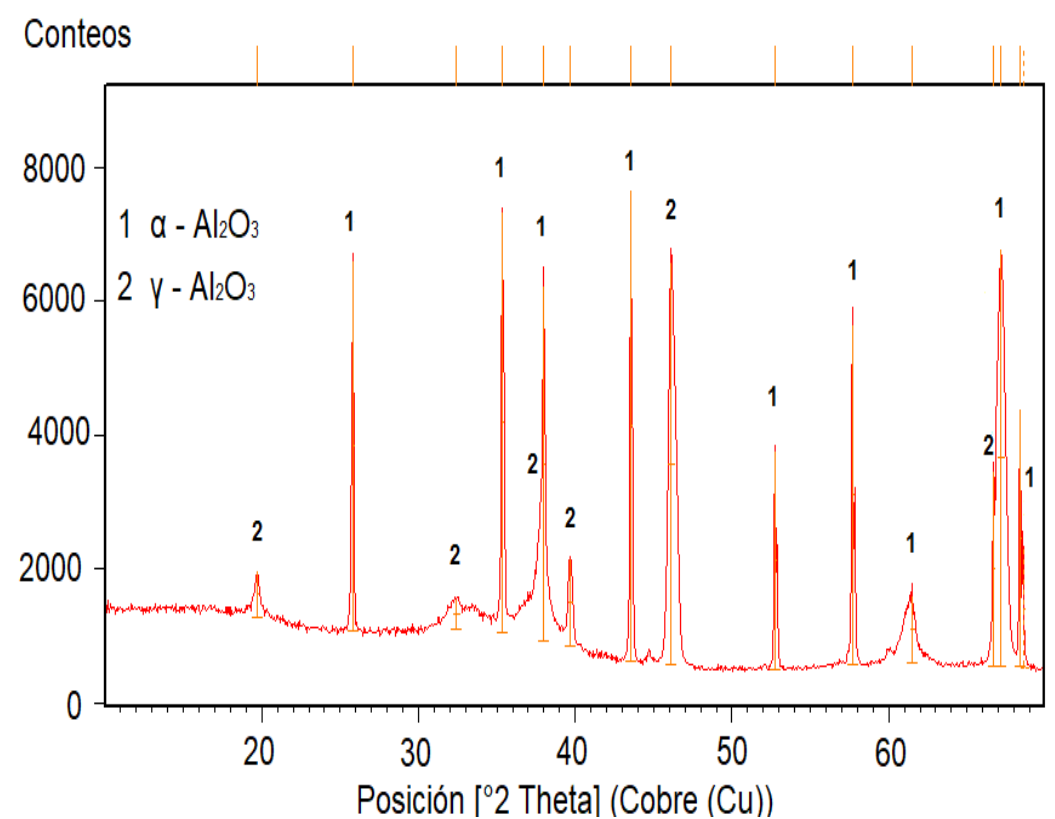

Fig. 5 Espectro de Difracción de Rayos X para la muestra M2-B. Fuente: autores.

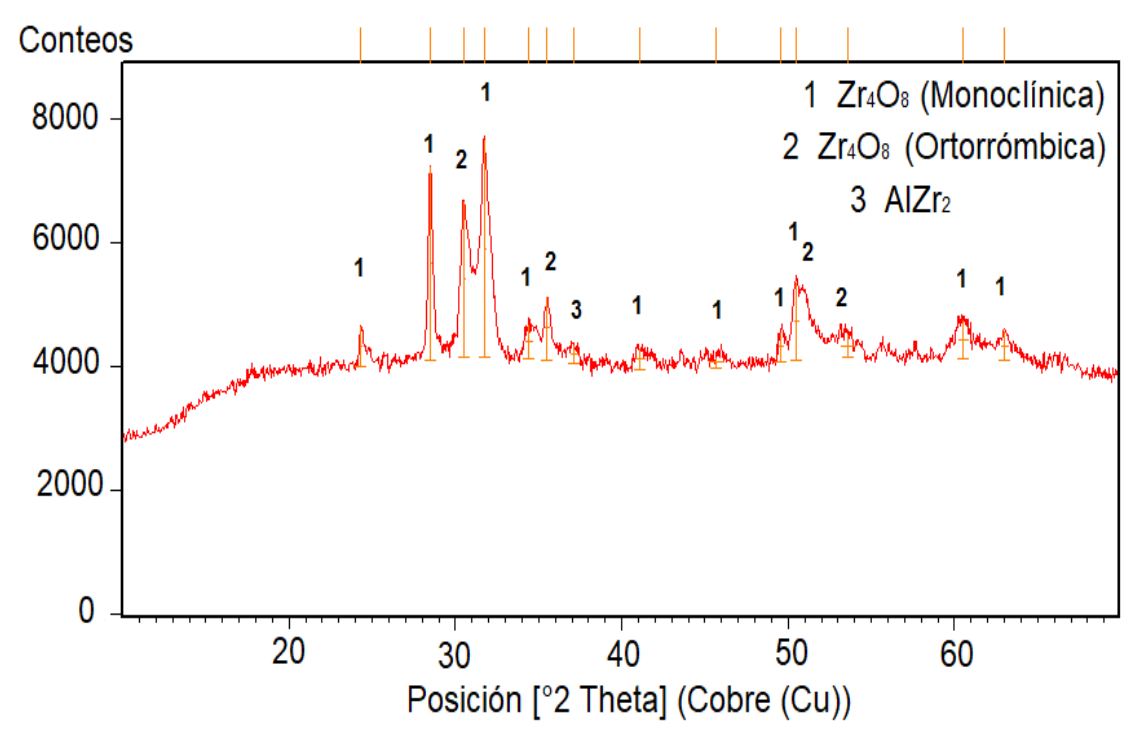

Fig. 6 Espectro de Difracción de Rayos X para la muestra M3-B. Fuente: autores

Tabla 6. Espesores de los recubrimientos depositados. Fuente autores

\begin{tabular}{ccc}
\hline Código de la muestra & Espesor capa base $(\mathrm{mm})$ & Espesor recubrimiento cerámico $(\mathrm{mm})$ \\
\hline M0-A & - & - \\
M0-B & $0,22 \pm 0,01$ & - \\
M1-A & - & $0,25 \pm 0,01$ \\
M1-B & $0,20 \pm 0,01$ & $0,35 \pm 0,01$ \\
M2-A & - & $0,10 \pm 0,03$ \\
M2-B & $0,22 \pm 0,01$ & $0,28 \pm 0,04$ \\
M3-A & - & $0,57 \pm 0,00$ \\
M3-B & $0,13 \pm 0,00$ & $0,24 \pm 0,01$ \\
\hline
\end{tabular}


Aislamiento térmico de tuberías de acero que transportan fluidos calientes a partir de recubrimientos elaborados mediante proyección térmica

De otro lado, en la muestra M3-B se identificaron los picos asociados al óxido de zirconio monoclínico $\left(\mathrm{Zr}_{4} \mathrm{O}_{8}\right)$ o Baddeleyita (Código de referencia COD 96-900-5834), marcados como $1, \mathrm{y}$ al ortorrómbico con el mismo nombre marcados como 2 (Código de referencia COD 96-900-5836). Algo característico observado en este análisis fue que los patrones utilizados para identificar las respectivas fases que mostraban un mejor ajuste en cuanto al ángulo adecuado en los picos más intensos, presentaban ligeros corrimientos hacia la izquierda del espectro. Estos corrimientos pueden asociarse a deformaciones que modifican las orientaciones de crecimiento de los cristales, causadas por esfuerzos compresivos generados en la intercara del cerámico con el sustrato o capa base durante la solidificación, y esfuerzos de tensión en la superficie generados también por la contracción térmica durante la solidificación. Una tercera fase marcada como 3 , corresponde a aluminatos de zirconio (AlZr2) (Código de referencia ICDD 00-48-1378).

\subsubsection{Determinación de la porosidad y análisis estructural}

En las Fig. 7, 8 y 9 se muestran las secciones transversales de los recubrimientos de las muestras M1-B, M2-B y M3-B.

De acuerdo con las Fig. 7, 8 y 9, se pudo establecer que el porcentaje de porosidad del recubrimiento de $\mathrm{Al}_{2} \mathrm{O}_{3}-\mathrm{TiO}_{2}$ es de 45,62 $\pm 5,24$, ver Fig. 7, mientras que el de los recubrimientos de $\mathrm{Al}_{2} \mathrm{O}_{3}$ y $\mathrm{Al}_{2} \mathrm{O}_{3} \approx 60 \%$ en peso de $\mathrm{ZrO} 2$ es de $65,49 \pm 5.81 \%$ y $38,29 \pm 6,51 \%$ respectivamente, ver Fig. 8 y 9. Si bien estos valores pueden ser producto de una sobreestimación a causa de arranques de material durante la etapa de pulido de las muestras para su análisis, conservan una proporción que puede ser tenida en cuenta al estar preparados de la misma manera, lo cual hace al sistema de $\mathrm{Al}_{2} \mathrm{O}_{3}$ el más poroso, seguido por el de
$\mathrm{Al}_{2} \mathrm{O}_{3}-\mathrm{TiO}_{2}$ y finalmente $\mathrm{Al}_{2} \mathrm{O}_{3}-\mathrm{ZrO}_{2}$ como el menos poroso.

El orden de porosidad determinado resulta consecuente con los puntos de fusión, de los valores de conductividad térmica y del tamaño de las partículas proyectadas, cuya sinergia conlleva a una mayor dificultad asociada a fundir completa o parcialmente las partículas. Es de anotar que las partículas de orden nanométrico y submicrométrico que constituyen el polvo de $\mathrm{Al}_{2} \mathrm{O}_{3} \approx 60 \%$ en peso de $\mathrm{ZrO}_{2}$ favorecen la fusión y por ende el recubrimiento obtenido es más compacto.

En las Fig. 7, 8 y 9 se puede observar que la rugosidad del sustrato permitió un buen acople de las capas base de cada sistema. Esto se evidencia en una interfaz ajustada y ceñida a la rugosidad del sustrato, sin desprendimientos y con espesores homogéneos en su longitud. Además, son evidentes algunas características físicas asociadas a la técnica empelada para la deposición de las capas. Entre ellas se destacan, por ejemplo, partículas parcialmente fundidas como en la Fig. 8 y líneas características del apilamiento de las partículas, o lamelles, como las señaladas en la Fig. 9. Estas características no comprometen la finalidad de aislamiento térmico de estas capas, ya que por el contrario algunas de ellas se encargan de generar porosidades y cavidades que ofrecen un medio discontinuo que dificulta la conducción del calor.

\subsection{Pruebas térmicas en estado transitorio}

Hechas las mediciones de temperatura al interior y al exterior de las muestras, los valores obtenidos se graficaron en función del tiempo para observar su comportamiento.

En la Fig. 10 se muestra que, en un primer tramo, hasta los 7 primeros minutos, la transferencia de calor por parte del aceite hacia los diferentes sistemas de aislamiento es más severa, de acuerdo con 


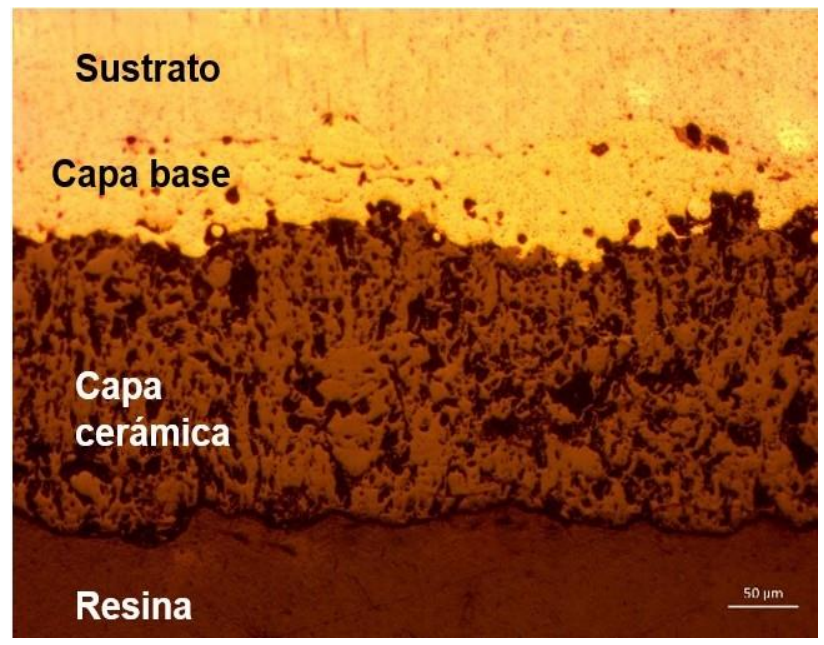

Fig. 7 Micrografía de la muestra M1-B. Fuente: autores.

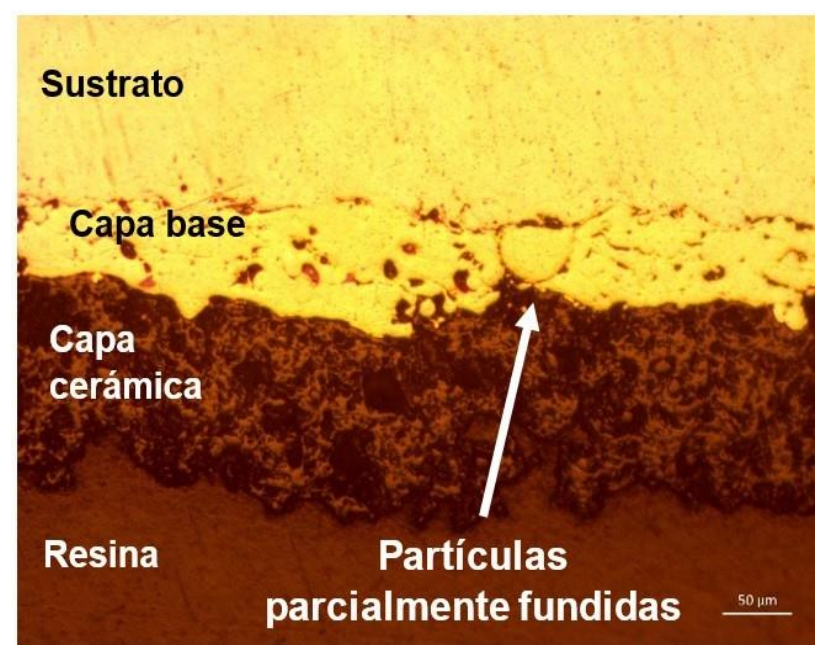

Fig. 8. Micrografía de la muestra M2-B. Fuente: autores.

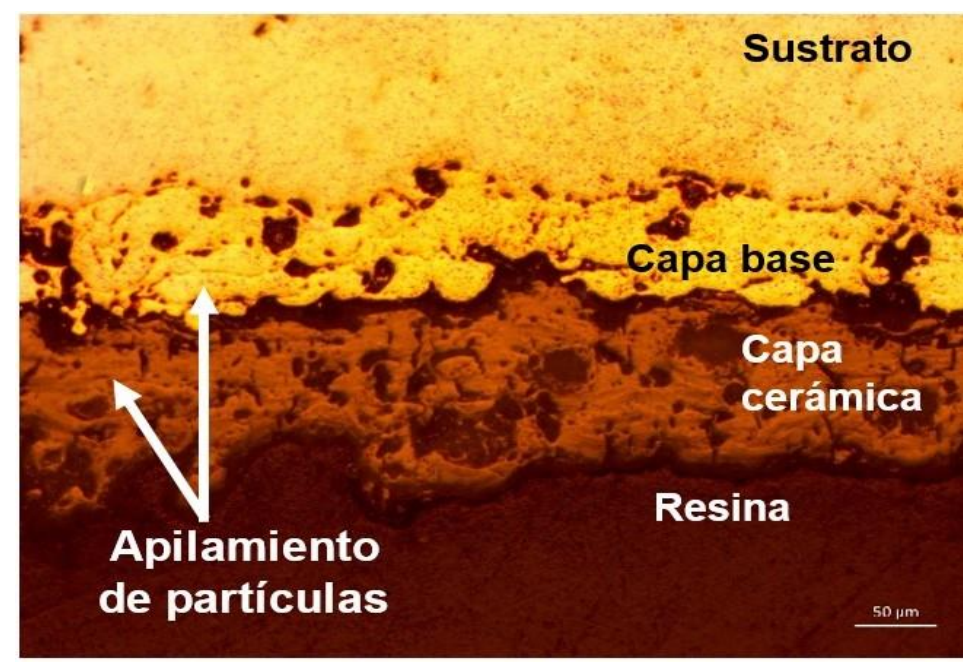

Fig. 9. Micrografía de la muestra M3-B. Fuente: autores.

la pendiente observada (línea roja punteada). Pasados diez minutos, inicia un segundo tramo con pendiente diferente, donde la transferencia de calor es menor y tiende a hacerse constante. A partir de los 20 minutos se apreció un tercer tramo, en el que las diferencias de temperatura entre un sistema u otro no son tan significativas. En la Tabla 7 puede observarse un compendio de las pendientes, denotadas como velocidades medias de enfriamiento para cada sistema.

El análisis de la temperatura exterior de las tuberías puede observarse en la Fig. 11. Es evidente que durante los primeros minutos del ensayo la temperatura incre- menta hasta alcanzar un pico de temperatura máxima a los cinco minutos, lo cual es asociado a la transferencia de calor predominantemente conductiva desde la pared interior a la exterior de la muestra, el cual es diferente para cada sistema de recubrimiento.

Pasados los cinco primeros minutos comienza una etapa de descenso de la temperatura exterior, asociada a un cambio del régimen que gobierna la transferencia de calor a uno convectivo, en el cual la tasa de extracción de calor por parte del aire que circunda el exterior de la tubería es mayor que la transferida por el aceite desde el interior del tubo. 
Aislamiento térmico de tuberías de acero que transportan fluidos calientes a partir de recubrimientos elaborados mediante proyección térmica

Tabla 7. Velocidad media de enfriamiento del aceite de silicona de los sistemas de aislamiento. Fuente: autores.

\begin{tabular}{|c|c|c|c|}
\hline & \multicolumn{3}{|c|}{ Fuente: autores. } \\
\hline & \multicolumn{3}{|c|}{ Velocidad media de enfriamiento $\left({ }^{\circ} \mathrm{C} / \mathrm{min}\right)$} \\
\hline & Tramo 1 & Tramo 2 & Tramo 3 \\
\hline Sistema & $0-7$ min & $7-20 \mathrm{~min}$ & $20-30 \mathrm{~min}$ \\
\hline $\mathrm{Al} 2 \mathrm{O} 3-\mathrm{TiO} 2$ & 5,9 & 2,8 & 1,4 \\
\hline Pintura & 6,1 & 2,5 & 1,4 \\
\hline $\mathrm{Ni}$ & 5,7 & 2,3 & 1,2 \\
\hline $\mathrm{Al} 2 \mathrm{O} 3-\mathrm{ZrO} 2$ & 5,4 & 2,5 & 1,3 \\
\hline Acero & 5,4 & 2,4 & 1,4 \\
\hline $\mathrm{Al} 2 \mathrm{O} 3-\mathrm{TiO} 2+\mathrm{Ni}$ & 5,3 & 2,5 & 1,2 \\
\hline $\mathrm{Al} 2 \mathrm{O} 3+\mathrm{Ni}$ & 6,0 & 2,2 & 1,2 \\
\hline $\mathrm{Al} 2 \mathrm{O} 3$ & 5,0 & 2,4 & 1,4 \\
\hline $\mathrm{Al} 2 \mathrm{O} 3-\mathrm{ZrO} 2+\mathrm{Ni}$ & 4,9 & 2,4 & 1,2 \\
\hline
\end{tabular}

Como es evidente, en la figura anterior, en el pico de máxima temperatura logrado a los cinco minutos de ensayo y donde prevalece el mecanismo conductivo de calor, la aplicación de una capa de aleación de níquel de tan solo 0,22 $\mathrm{mm}$ (probeta M0-B) reduce la temperatura exterior del tubo de acero (muestra M0-A) de 73,9 a $72,5{ }^{\circ} \mathrm{C}$, lo que indica que la capa base a pesar de estar constituida principalmente por níquel, cuyo coeficiente de conducción $(\mathrm{K}=60,7 \mathrm{~W} / \mathrm{m}-\mathrm{K}[1])$ es superior al del acero al carbono de composición similar a la de los tubos (K entre $27 \mathrm{y} 52 \mathrm{~W} / \mathrm{m}-\mathrm{K}$ [1]) utilizados como sustrato, logra reducir la temperatura exterior, puesto que actúa como una resistencia adicional que se opone al paso de calor desde la pared interna de la tubería hacia el exterior de ella. Así mismo, al remplazar la capa base por un recubrimiento de alúmina de tan solo $0,1 \mathrm{~mm}$ (muestra M2-A), la temperatura del tubo de acero desciende de 73,9 a $68,1{ }^{\circ} \mathrm{C}$, lo que indica que esta capa cerámica a pesar de tener un coeficiente de conductividad térmica (K entre 32-46 W/m-K [1]) del mismo orden de magnitud que el del acero también actúa como una resistencia al flujo de calor, la cual es más eficiente que la resistencia ofrecida por una capa de níquel que le dobla en espesor, dado su menor coeficiente de conducción de calor. La utilización de la capa base previa a la capa de alúmina (muestra M2-B) reduce aún más la temperatura exterior del tubo de 73,9 a $65,6{ }^{\circ} \mathrm{C}$, lo cual es favorecido además por el aumento en el espesor de la capa de alúmina.

En el tubo recubierto solo con la capa de $\mathrm{Al}_{2} \mathrm{O}_{3} \approx 40 \%$ en peso de $\mathrm{TiO}_{2}$ (muestra M1A), la temperatura exterior desciende de 73,9 a $64,9{ }^{\circ} \mathrm{C}$ respecto al tubo sin recubrimiento. La menor temperatura exterior respecto a las muestras M0-B y M2-A, con capa base de aleación de níquel de espesor similar $(0,22 \mathrm{~mm}$ para la capa base vs 0,25 mm para la capa cerámica) y con capa de alúmina sin capa base (de menor espesor $0,1 \mathrm{~mm}$, pero mayor cantidad de poros), se debe a que el coeficiente de conductividad térmica de la capa cerámica $(1,5 \mathrm{~W} / \mathrm{m}-\mathrm{K}$ [1]) es muy inferior al de la capa base y al de la capa de alúmina (60,7 y entre 32 y 46 $\mathrm{W} / \mathrm{m}-\mathrm{K}$ respectivamente [1]).

A pesar de la diferencia tan significativa entre los coeficientes de conducción de calor del recubrimiento de alúmina (muestra M2-A) y del de $\mathrm{Al}_{2} \mathrm{O}_{3} \approx 40 \%$ en peso de $\mathrm{TiO}_{2}$ (muestra M1-A) y el mayor espesor de esta última capa, la reducción en temperatura exterior de la tubería lograda con una capa de alúmina de $0,1 \mathrm{~mm}$ de espesor (muestra M2-A) frente a la alcanzada con la capa de $\mathrm{Al}_{2} \mathrm{O}_{3} \approx 40 \%$ en peso de $\mathrm{TiO}_{2}$ de $0,25 \mathrm{~mm}$ de espesor (muestra M1-A) no es tan significativa $\left(68,1\right.$ y $64,9{ }^{\circ} \mathrm{C}$ respectivamente), lo cual indicaría que la mayor porosidad en el recubrimiento de alúmina $(65,49 \%)$, frente a la de la capa de $\mathrm{Al}_{2} \mathrm{O}_{3} \approx 40 \%$ en peso de $\mathrm{TiO}_{2}$ (45,62 \%) contribuye a aumentar su resistencia al flujo de calor desde el interior del ducto. 
Aislamiento térmico de tuberías de acero que transportan fluidos calientes a partir de recubrimientos elaborados mediante proyección térmica

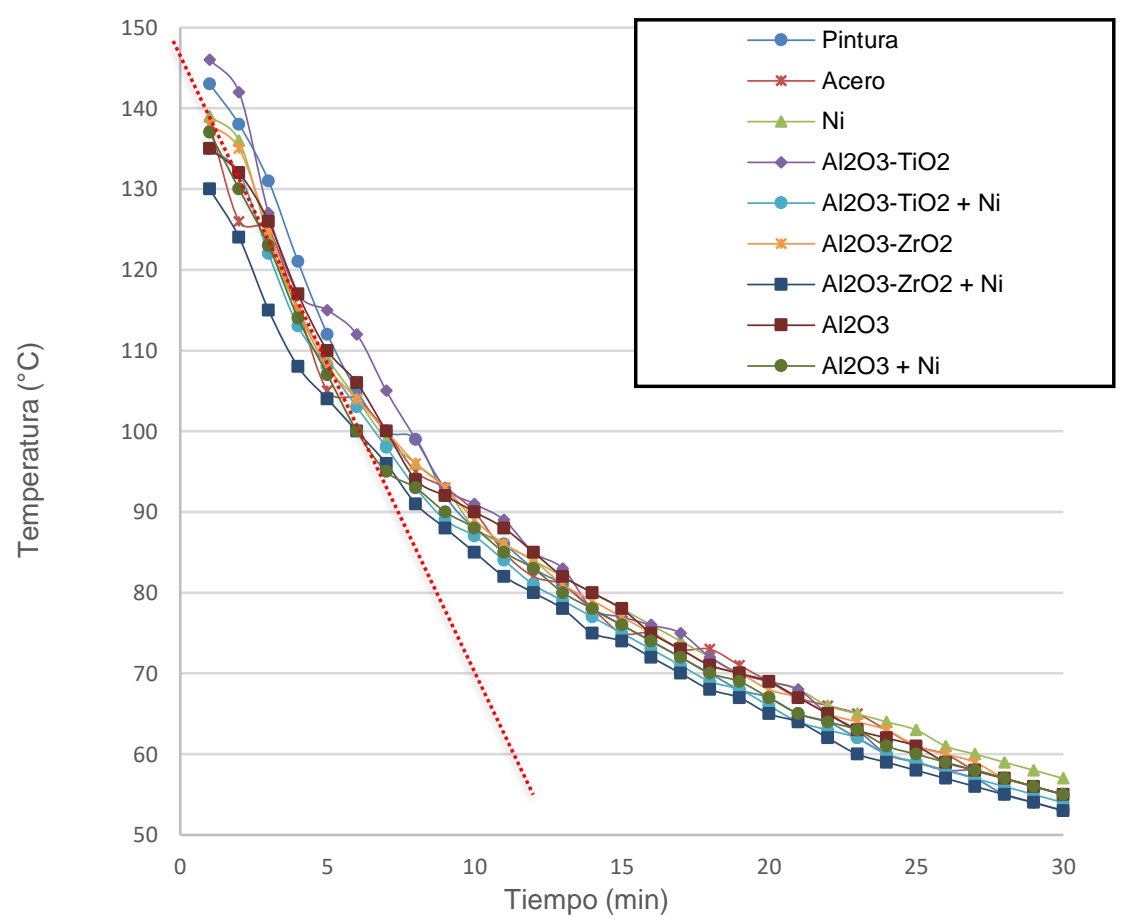

Fig.10. Variación de la temperatura del aceite de silicona para las diferentes probetas. Fuente: autores.

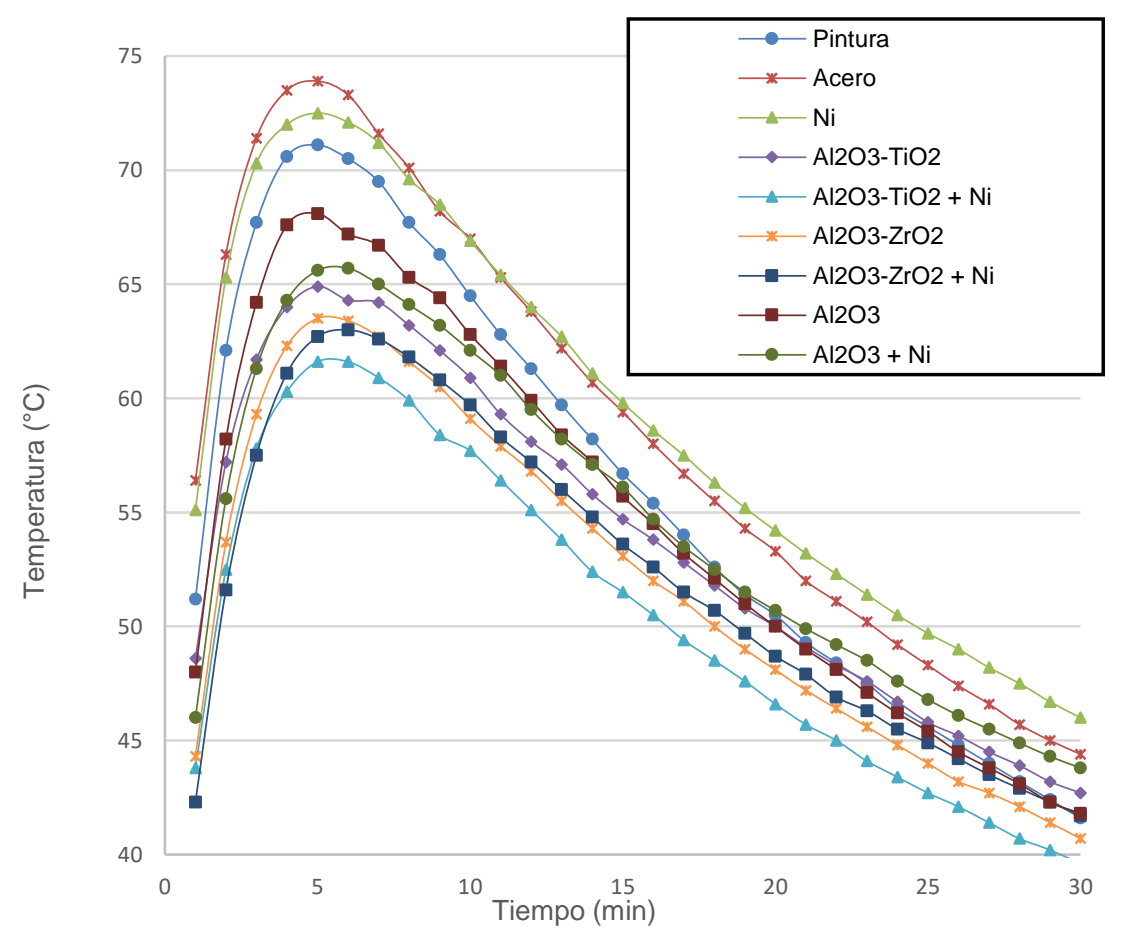

Fig.11. Velocidad media de enfriamiento del aceite de silicona de los sistemas de aislamiento. Fuente: autores. 
Aislamiento térmico de tuberías de acero que transportan fluidos calientes a partir de recubrimientos elaborados mediante proyección térmica

Por su parte, el recubrimiento de $\mathrm{Al}_{2} \mathrm{O}_{3} \approx 40 \%$ en peso de $\mathrm{ZrO}_{2}$ depositado sin capa base (muestra M3-A) pero con un espesor grueso de 0,57 $\mathrm{mm}$ logra una reducción en la temperatura exterior del tubo de 73,9 a $63,5^{\circ} \mathrm{C}$, la cual es mayor que la lograda en las muestras descritas anteriormente, incluso que en la lograda con el recubrimiento de $\mathrm{Al}_{2} \mathrm{O}_{3} \approx 40 \%$ en peso de $\mathrm{TiO} 2$, a pesar de su mayor coeficiente de conducción térmica $(21,6$ frente a $1,5 \mathrm{~W} / \mathrm{m}$ $\mathrm{K}$ [1]), lo que indica que el espesor de la capa del recubrimiento de $\mathrm{Al}_{2} \mathrm{O}_{3} \approx 60 \%$ en peso de $\mathrm{ZrO}_{2}$ contribuye a aumentar su resistencia al flujo de calor desde el interior del ducto.

Como es evidente, en la figura anterior, en el pico de máxima temperatura logrado a los cinco minutos de ensayo y donde prevalece el mecanismo conductivo de calor, la aplicación de una capa de aleación de níquel de tan solo 0,22 $\mathrm{mm}$ (probeta M0-B) reduce la temperatura exterior del tubo de acero (muestra M0-A) de 73,9 a $72,5{ }^{\circ} \mathrm{C}$, lo que indica que la capa base a pesar de estar constituida principalmente por níquel, cuyo coeficiente de conducción ( $\mathrm{K}=60,7 \mathrm{~W} / \mathrm{m}-\mathrm{K}$ [1]) es superior al del acero al carbono de composición similar a la de los tubos (K entre 27y $52 \mathrm{~W} / \mathrm{m}-\mathrm{K}$ [1]) utilizados como sustrato, logra reducir la temperatura exterior, puesto que actúa como una resistencia adicional que se opone al paso de calor desde la pared interna de la tubería hacia el exterior de ella. Así mismo, al remplazar la capa base por un recubrimiento de alúmina de tan solo $0,1 \mathrm{~mm}$ (muestra M2-A), la temperatura del tubo de acero desciende de 73,9 a $68,1^{\circ} \mathrm{C}$, lo que indica que esta capa cerámica a pesar de tener un coeficiente de conductividad térmica ( $\mathrm{K}$ entre $32-46 \mathrm{~W} / \mathrm{m}-\mathrm{K}$ [1]) del mismo orden de magnitud que el del acero también actúa como una resistencia al flujo de calor, la cual es más eficiente que la resistencia ofrecida por una capa de níquel que le dobla en espesor, dado su menor coeficiente de conducción de calor. La utiliza- ción de la capa base previa a la capa de alúmina (muestra M2-B) reduce aún más la temperatura exterior del tubo de 73,9 a $65,6{ }^{\circ} \mathrm{C}$, lo cual es favorecido además por el aumento en el espesor de la capa de alúmina.

En el tubo recubierto solo con la capa de $\mathrm{Al}_{2} \mathrm{O}_{3} \approx 40 \%$ en peso de $\mathrm{TiO}_{2}$ (muestra M1A), la temperatura exterior desciende de 73,9 a $64,9{ }^{\circ} \mathrm{C}$ respecto al tubo sin recubrimiento. La menor temperatura exterior respecto a las muestras $\mathrm{M} 0-\mathrm{B}$ y $\mathrm{M} 2-\mathrm{A}$, con capa base de aleación de níquel de espesor similar $(0,22 \mathrm{~mm}$ para la capa base vs 0,25 mm para la capa cerámica) y con capa de alúmina sin capa base (de menor espesor $0,1 \mathrm{~mm}$ pero mayor cantidad de poros), se debe a que el coeficiente de conductividad térmica de la capa cerámica $(1,5 \mathrm{~W} / \mathrm{m}-\mathrm{K}$ [1]) es muy inferior al de la capa base y al de la capa de alúmina (60,7 y entre 32 y 46 $\mathrm{W} / \mathrm{m}-\mathrm{K}$ respectivamente [1]).

A pesar de la diferencia tan significativa entre los coeficientes de conducción de calor del recubrimiento de alúmina (muestra M2-A) y del de $\mathrm{Al}_{2} \mathrm{O}_{3} \approx 40 \%$ en peso de $\mathrm{TiO}_{2}$ (muestra M1-A) y el mayor espesor de esta última capa, la reducción en temperatura exterior de la tubería lograda con una capa de alúmina de $0,1 \mathrm{~mm}$ de espesor (muestra M2-A) frente a la alcanzada con la capa de $\mathrm{Al}_{2} \mathrm{O}_{3} \approx 40 \%$ en peso de $\mathrm{TiO}_{2}$ de 0,25 mm de espesor (muestra M1-A) no es tan significativa $\left(68,1\right.$ y $64,9{ }^{\circ} \mathrm{C}$ respectivamente), lo cual indicaría que la mayor porosidad en el recubrimiento de alúmina $(65,49 \%)$, frente a la de la capa de $\mathrm{Al}_{2} \mathrm{O}_{3} \approx 40 \%$ en peso de $\mathrm{TiO}_{2}$ (45,62 \%) contribuye a aumentar su resistencia al flujo de calor desde el interior del ducto.

Por su parte, el recubrimiento de $\mathrm{Al}_{2} \mathrm{O}_{3} \approx 40 \%$ en peso de $\mathrm{ZrO}_{2}$ depositado sin capa base (muestra M3-A) pero con un espesor grueso de 0,57 $\mathrm{mm}$ logra una reducción en la temperatura exterior del tubo de 73,9 a $63,5^{\circ} \mathrm{C}$, la cual es mayor que la lograda en las muestras descritas anteriormente, incluso que en la lograda con el 
Aislamiento térmico de tuberías de acero que transportan fluidos calientes a partir de recubrimientos elaborados mediante proyección térmica

recubrimiento de $\mathrm{Al}_{2} \mathrm{O}_{3} \approx 40 \%$ en peso de $\mathrm{TiO} 2$, a pesar de su mayor coeficiente de conducción térmica $(21,6$ frente a $1,5 \mathrm{~W} / \mathrm{m}$ $\mathrm{K}$ [1]), lo que indica que el espesor de la capa del recubrimiento de $\mathrm{Al}_{2} \mathrm{O}_{3} \approx 60 \%$ en peso de $\mathrm{ZrO}_{2}$ contribuye a aumentar su resistencia al flujo de calor desde el interior del ducto.

Finalmente, la muestra que alcanza la menor temperatura en la cara externa del tubo es la recubierta con la capa cerámica de $\mathrm{Al}_{2} \mathrm{O}_{3} \approx 40 \%$ en peso de $\mathrm{TiO}_{2}$ con capa base de aleación de níquel (muestra M1-B), seguida de la del recubrimiento de $\mathrm{Al}_{2} \mathrm{O}_{3} \approx 60 \%$ en peso de $\mathrm{ZrO}_{2}$ depositada sobre una capa base de níquel. Este comportamiento se asocia a la menor conductividad térmica de estos dos cerámicos (1,5 y $21,6 \mathrm{~W} / \mathrm{m}-\mathrm{K}$ [1] respectivamente) y que la capa base de aleación de níquel, a pesar de presentar una alta transferencia de calor se comporta como una barrera que puede reducir ligeramente la temperatura proveniente de la pared interna de tubo de acero al carbono en su paso hacia el recubrimiento cerámico.

\section{CONCLUSIONES}

Se evidenció que la capa base de níquel, que inicialmente se planteó como una alternativa para contrarrestar el efecto de las dilataciones térmicas y salvaguardar la integridad del recubrimiento, permite disminuir el escape de calor en aquellos procesos donde el mecanismo conductivo sea preponderante.

De acuerdo con los resultados de las pruebas térmicas realizadas en un estado transitorio, se pudo concluir que en los procesos de transferencia de calor gobernados por la conductividad térmica, el sistema metal-cerámico de $\mathrm{Al}_{2} \mathrm{O}_{3} \approx 40 \%$ en peso de $\mathrm{TiO}_{2}$ depositado sobre una capa de níquel es el que presenta la mejor condición de aislamiento térmico, la cual puede ser favorecida por el aumento en el espesor de la capa tanto cerámica como de níquel y por la inclusión de porosidades en la estructura del recubrimiento. Lo anterior, gracias al bajo coeficiente de conductividad térmica que posee este material y a la mayor dificultad que tiene el calor para ser conducido a medida que aumenta el espesor y la porosidad de los recubrimientos.

\section{AGRADECIMIENTOS}

Los autores agradecen al Fondo de Apoyo a los Trabajos de Grado de la Facultad de Ingeniería de la Universidad de Antioquia y al CODI por la financiación del proyecto 2016/1-04-TG-C.

\section{REFERENCIAS}

[1] MatWeb, "Online Materials Information Resource - MatWeb”, 2016. [En línea]. Disponible en: http://www.matweb.com/. [Accedido: 03-ene2016].

[2] G. Latorre y F. Vargas, "Materiales compuestos reforzados con cerámicos amorfos para aplicaciones a altas temperaturas", CT\&F Ciencia, Tecnol. y Futur., vol. 3, pp. 175-188, 2009.

[3] G. Latorre y F. Vargas, "Nuevos Materiales, Materiales Compuestos, Tratamiento de superficies", Mater. Ing. - Ecopetrol, vol. 1, pp. 103-119, 2007.

[4] Y. Başoğul y A. Keçebaş, "Economic and environmental impacts of insulation in district heating pipelines", Energy, vol. 36, no. 10, pp. 61566164 , oct. 2011.

[5] A. Bahadori y H. B. Vuthaluru, "A simple correlation for estimation of economic thickness of thermal insulation for process piping and equipment", Appl. Therm. Eng., vol. 30, no. 2-3, pp. 254-259, feb. 2010.

[6] J. Smith, William \& Hashemi, Fundamentos de la ciencia e ingenieria de materiales, 4.a ed. México, 2006.

[7] A. T. J. Verbeek, "Plasma Sprayed Thermal Barrier Coatings: Production, Characterization and Testing", Technische Universiteit Eindhoven, 1992.

[8] S. Bose, "Chapter 7 - THERMAL BARRIER COATINGS (TBCs)", en High Temperature Coatings, 2007, pp. 155-232.

[9] I. D. Utu, G. Marginean, I. Hulka, V. A. Serban, y D. Cristea, "Properties of the thermally sprayed Al2O3-TiO2 coatings deposited on tita- 
Aislamiento térmico de tuberías de acero que transportan fluidos calientes a partir de recubrimientos elaborados mediante proyección térmica

nium substrate", Int. J. Refract. Met. Hard Mater., vol. 51, pp. 118-123, jul. 2015.

[10] W. R. Chen, X. Wu, B. R. Marple, R. S. Lima, y P. C. Patnaik, "Pre-oxidation and TGO growth behaviour of an air-plasma-sprayed thermal barrier coating", Surf. Coatings Technol., vol. 202, pp. 3787-3796, 2008.

[11] E. Restrepo, F. Vargas, M. López, R. Cardona, y G. Duarte, "Elaboración de recubrimientos cerámicos mediante proyección térmica por combustión a partir de residuos sólidos industriales Elaboration of ceramic coatings by flame spraying process from industrial solid waste", Rev. Mater., vol. 17 (4), pp. 1176-1185, 2012.

[12] F. Vargas y G. Latorre, "Materiales de Ingeniería", Ecopetrol, vol. 1, pp. 131-160, 2007.

[13] M. Mellali, A. Grimaud, A. C. Leger, P. Fauchais, y J. Lu, "Alumina grit blasting parameters for surface preparation in the plasma spraying operation", J. Therm. Spray Technol., vol. 6, n.o 2, pp. 217-227, jun. 1997.

[14] "Thermodynamic and Kinetic Data", en CRC Materials Science and Engineering Handbook, Third Edition, CRC Press, 2000.

[15] K. A. Habib, J. J. Saura, C. Ferrer, M. S. Damra, E. Giménez, y L. Cabedo, "Comparison of flame sprayed $\mathrm{Al} 2 \mathrm{O} 3 / \mathrm{TiO} 2$ coatings: Their microstruc- ture, mechanical properties and tribology behavior", Surf. Coatings Technol., vol. 201, n.o 3-4, pp. 1436-1443, oct. 2006.

[16] W. D. Callister, Materials Science and Engineering: An Introduction, 7.a ed. Estados Unidos: John Wiley \& Sons, Inc., 2007.

[17]A. G. González Hernández, "Estudio De La Influencia De Las Propiedades Físicas Y Mecánicas En El Comportamiento Tribológico De Recubrimientos Duros Para Herramientas De Corte Y Procesamiento De Polietileno", Universidad de Antioquia, 2008.

[18] J. E. Rodríguez, F. Vargas, E. López, E. Restrepo, and J. F. Gil, "Mejoramiento de la estructura de los recubrimientos de Al2o3-43\% Tio2 elaborados mediante la técnica de proyección térmica por llama oxiacetilénica a partir de los parámetros de proyección," Rev. Colomb. Mater., no. 5, pp. 120-126, 2014.

[19]W. Conshohocken, "ASTM E1920 - Standard Guide for Metallographic Preparation of Thermal Sprayed Coatings", ASM Int., vol. 3, n.o Reapproved 2014, pp. 1-5, 2014.

[20] P. S. Santos, H. S. Santos, y S. P. Toledo, "Standard transition aluminas. Electron microscopy studies", Mater. Res., vol. 3, n.o 4, pp. 104-114, 2000. 